OPEN ACCESS

Edited by:

Jiehui Jiang,

Shanghai University, China

Reviewed by:

Richard Camicioli,

University of Alberta, Canada

Lirong Jin,

Zhongshan Hospital, China

Jong-Min Kim,

Seoul National University Bundang Hospital, South Korea

Paolo Caffarra,

University of Parma, Italy

*Correspondence:

Huifang Shang

hfshang2002@126.com

Specialty section:

This article was submitted to

Parkinson's Disease

and Aging-Related Movement

Disorders,

a section of the journal

Frontiers in Aging Neuroscience

Received: 03 October 2021

Accepted: 03 January 2022

Published: 25 January 2022

Citation:

Hou Y and Shang H (2022)

Magnetic Resonance Imaging

Markers for Cognitive Impairment

in Parkinson's Disease: Current View.

Front. Aging Neurosci. 14:788846.

doi: 10.3389/fnagi.2022.788846

\section{Magnetic Resonance Imaging Markers for Cognitive Impairment in Parkinson's Disease: Current View}

\author{
Yanbing Hou and Huifang Shang* \\ Laboratory of Neurodegenerative Disorders, Department of Neurology, Rare Diseases Center, National Clinical Research \\ Center for Geriatrics, West China Hospital, Sichuan University, Chengdu, China
}

Cognitive impairment $(\mathrm{Cl})$ ranging from mild cognitive impairment $(\mathrm{MCl})$ to dementia is a common and disturbing complication in patients with Parkinson's disease (PD). Numerous studies have focused on neuropathological mechanisms underlying $\mathrm{Cl}$ in $\mathrm{PD}$, along with the identification of specific biomarkers for $\mathrm{Cl}$. Magnetic resonance imaging (MRI), a promising method, has been adopted to examine the changes in the brain and identify the candidate biomarkers associated with $\mathrm{Cl}$. In this review, we have summarized the potential biomarkers for $\mathrm{Cl}$ in PD which have been identified through multi-modal MRI studies. Structural MRI technology is widely used in biomarker research. Specific patterns of gray matter atrophy are promising predictors of the evolution of $\mathrm{Cl}$ in patients with PD. Moreover, other MRI techniques, such as MRI related to small-vessel disease, neuromelanin-sensitive MRI, quantitative susceptibility mapping, MR diffusion imaging, MRI related to cerebrovascular abnormality, restingstate functional $\mathrm{MRI}$, and proton magnetic resonance spectroscopy, can provide imaging features with a good degree of prediction for $\mathrm{Cl}$. In the future, novel combined biomarkers should be developed using the recognized analysis tools and predictive algorithms in both cross-sectional and longitudinal studies.

Keywords: Parkinson's disease, magnetic resonance imaging, biomarker, mild cognitive impairment, dementia

\section{INTRODUCTION}

Parkinson's disease (PD) is becoming the fastest-growing neurological disorder. In 2016, globally, approximately 6.1 million people, suffered from PD, and this incidence is further expected to double to more than 12 million by 2040. Cardinal motor symptoms of PD are easy to identify and can be controlled by anti-PD medicine. However, various non-motor symptoms, including cognitive impairment (CI), mood disorders, neuropsychic symptoms, autonomic dysfunction, and pain, greatly aggravate the disability of the affected individuals (Dorsey et al., 2018; Collaborators, 2019; Bloem et al., 2021). CI is a common non-motor symptom that progresses from normal cognition to mild cognitive impairment (MCI), and eventually dementia. Dementia is a common disorder occurring in advanced stages of PD, and MCI, the pre-dementia stage of CI, has certainly attracted great attention in recent years. To promote uniformity in clinical and research applications on 
cognition in $\mathrm{PD}$, clinical diagnostic criteria for different stages of CI (MCI and dementia) have been delineated by a panel of experts from the Movement Disorder Society (MDS) Task Force (Emre et al., 2007; Litvan et al., 2012).

The diagnostic criteria of PD patients with MCI (PDMCI) are as follows: (1) a diagnosis of PD; (2) a gradual decline of the cognitive ability in the level I category based on a brief cognitive assessment or in the level II category based on a more comprehensive neuropsychological assessment including at least two tests for each of five cognitive domains (attention, executive function, language, memory, and visuospatial function); and (3) complete functional independence. Impairment in neuropsychological tests is defined per one to two standard deviations (SDs) below the appropriate norms, a significant decrease in the serial cognitive tests, or a significant decrease from the estimated premorbid levels. The PD patient with MCI according to the level II criteria shows impairment in at least two tests within one single cognitive domain or different cognitive domains (Litvan et al., 2012). Following are the diagnostic criteria of $\mathrm{PD}$ patients with dementia (PDD): (1) a diagnosis of PD; (2) progressive impairment in more than one cognitive domain or a significant decrease from premorbid levels; and (3) impaired functional independence (Emre et al., 2007). Based on the MDS Task Force Level II criteria, recent studies report that the frequency of PD-MCI is between 20\% and 35\% in newly diagnosed PD patients (Broeders et al., 2013; Pedersen et al., 2017). Moreover, according to the clinical criteria, a recent meta-analysis comprising of 41 studies reported that the pooled PD-MCI prevalence is $40 \%$ in a total sample of 7,053 PD patients (Baiano et al., 2020). In a prospective population-based study, among the newly diagnosed PD patients with normal cognition at baseline, the cumulative incidence of PD-MCI is $9.9 \%$ after 1 year, 23.2\% after 3 years, and 28.9\% after 5 years of follow-up (Pedersen et al., 2017). Patients with PD-MCI are more likely to eventually develop PDD, and 59.1\% of patients with persistent PD-MCI within 1 year develop PDD (Pedersen et al., 2017). The point prevalence of PDD is close to $30 \%$ in a PD cohort, and at least $75 \%$ of PD patients who survived for more than 10 years are more likely to develop dementia (Aarsland and Kurz, 2010).

Although dementia is a common problem in patients with advanced $\mathrm{PD}$, there are great differences in the rate of cognitive decline and the time of dementia onset in PD patients. Thus, effective markers that can accurately quantify pathological changes are needed to identify $\mathrm{PD}$ patients at high risk of developing $\mathrm{CI}$, to predict the progression of cognitive dysfunction, and for ease of their availability. In the last few years, magnetic resonance imaging (MRI) technology, an attractive option, has improved the understanding of the pathophysiology of CI in PD patients, along with establishing potential biomarkers. These biomarkers for cognitive dysfunction in PD were reviewed based on a PubMed search for peer-reviewed articles published between January 2011 and August 2021 using the search terms "marker" OR "biomarker" AND "Parkinson's disease" OR "Parkinson" OR "Parkinson's disease" AND "mild cognitive impairment" OR "MCI" OR "Parkinson's disease dementia" OR "dementia" OR "cognitive impairment” AND “MRI” OR “magnetic resonance imaging”. This review provides an update on the recent advances in the emerging role and development of MRI markers for cognitive dysfunction in PD.

\section{STRUCTURAL MAGNETIC RESONANCE IMAGING}

Most of the studies in recent years acquired structural MR images using a $3.0 \mathrm{~T}$ scanner, and grey matter (GM) abnormalities are extensively examined using either voxel-based morphometry (VBM) or surface-based analyses. Automated and unbiased VBM analysis can be used to estimate the GM volume or concentration differences between groups at the whole-brain level, thus, it has been widely used to quantify regional GM alterations in PD patients with CI. Cortical thickness, the distance between white and pial surfaces, is a more sensitive structural MRI-based measurement to detect early GM changes. The details of imaging methods are presented in Table 1. The region of interest (ROI) approach has been used to analyze both cortical and subcortical changes based on an a priori hypothesis. Summary findings from structural MRI correlated with cognitive dysfunction in PD are showed in Table 2.

\section{Cross-Sectional MR Studies}

Cross-sectional results on CI-related GM changes in PD patients remain heterogeneous, and three meta-analyses were conducted to identify the most consistent GM alterations associated with PD-CI (Xu et al., 2016; Mihaescu et al., 2019; Zheng et al., 2019). A meta-analysis study in 2016, using five studies compared 92 PD-MCI patients with 192 PD without CI (PD-NCI) patients, and from ten studies, compared 168 PDD patients with $233 \mathrm{PD}$ without dementia (PD-ND) patients. The authors have reported significantly reduced GM volume in the left superior frontal gyrus (SFG), left superior temporal lobe (STL), and left insula in the PD-MCI group, and in the left SFG, and bilateral STL extending to the hippocampus in the PDD group (Xu et al., 2016). A meta-analysis study in 2019 compared 405 PD-MCI patients with 559 PD-NCI patients from fifteen studies, and compared 178 PDD patients with 278 PD-ND patients from eleven studies. A significantly higher GM atrophy in bilateral dorsolateral prefrontal cortex, left angular gyrus, right supramarginal gyrus, left insula, and midcingulate cortex in the PD-MCI group, and bilateral insula and right hippocampus in the PDD group was reported (Mihaescu et al., 2019). Another meta-analysis study in 2019 consisting of 504 PD-MCI patients and 554 PD-NCI patients from twenty studies reported a drastically remarkable GM atrophy in the left anterior insula extending to the inferior frontal gyrus, and orbital part in the PD-MCI group (Zheng et al., 2019). GM atrophy in the insula and frontal-limbictemporal regions are robust features of CI in PD, and unilateralto-bilateral development of structural atrophy is a potential indicator for progression of PD-MCI to PDD. In particular, several studies attempted to analyze GM changes across various stages of cognitive decline by comparing a prospective sample of PD-NCI, PD-MCI, PDD patients, and healthy subjects (HCs). 
TABLE 1 | Imaging characteristics of magnetic resonance imaging studies in Parkinson's disease and cognitive impairment.

\begin{tabular}{|c|c|c|c|c|}
\hline Modality & MR sequence & Core software/method & Analysis variables & Advantages/disadvantages \\
\hline \multirow[t]{2}{*}{ Structural imaging } & $\begin{array}{l}\text { T1-TFE sequence/3D } \\
\text { SPGR/MPRAGE }\end{array}$ & $\begin{array}{l}\text { SPM (http://www.fil.ion.ucl. } \\
\text { ac.uk/spm)/ } \\
\text { Freesurfer (http://surfer.nmr. } \\
\text { mgh.harvard.edu) }\end{array}$ & $\begin{array}{l}\text { Voxel-based } \\
\text { morphometry (VBM) }\end{array}$ & $\begin{array}{l}\text { It can estimate the gray matter (GM) } \\
\text { volume or concentration alterations at } \\
\text { the whole-brain level. }\end{array}$ \\
\hline & & $\begin{array}{l}\text { Freesurfer (http://surfer.nmr. } \\
\text { mgh.harvard.edu) }\end{array}$ & Cortical thickness (CTh) & $\begin{array}{l}\text { A more sensitive structural MRI-based } \\
\text { measurement to detect early GM } \\
\text { changes. }\end{array}$ \\
\hline \multirow[t]{3}{*}{$\begin{array}{l}\text { MRI related to } \\
\text { small-vessel disease }\end{array}$} & $\begin{array}{l}\text { T2-weighted fast } \\
\text { spin-echo } \\
\text { sequence/FLAIR }\end{array}$ & $\begin{array}{l}\text { Fazekas visual rating scale } \\
\text { (Scheltens et al., 1993) }\end{array}$ & $\begin{array}{l}\text { White matter } \\
\text { hyperintensity }(\mathrm{WMH})\end{array}$ & $\begin{array}{l}\text { It can reflect pathological white matter } \\
(\mathrm{WM}) \text { tissue ischemia. } \\
\text { It can be assessed on the basis of size } \\
\text { and quantity of lesions in a given } \\
\text { neuroanatomical location. }\end{array}$ \\
\hline & & $\begin{array}{l}\text { Automated routines (de } \\
\text { Boer et al., 2009; Bohnen } \\
\text { and Albin, 2011) }\end{array}$ & WMH & $\begin{array}{l}\text { Quantitative assessment of WML } \\
\text { burden may be particularly } \\
\text { advantageous for assessing changes } \\
\text { over time compared with the visual } \\
\text { grading. }\end{array}$ \\
\hline & & $\begin{array}{l}\text { Standards for Reporting } \\
\text { Vascular Changes on } \\
\text { Neuroimaging criteria } \\
\text { (Wardlaw et al., 2013) }\end{array}$ & $\begin{array}{l}\text { Perivascular space } \\
\text { (PVS) }\end{array}$ & $\begin{array}{l}\text { It is called the Virchow-Robin spaces } \\
\text { and is piallined interstitial fluid-filled } \\
\text { space surrounding the penetrating } \\
\text { vessels. }\end{array}$ \\
\hline $\begin{array}{l}\text { Neuromelanin-sensitive } \\
\text { MRI }\end{array}$ & $\begin{array}{l}\text { T1-weighted FSE } \\
\text { sequence }\end{array}$ & $\begin{array}{l}\text { Semi-automated analysis } \\
\text { (Sulzer et al., 2018). }\end{array}$ & Signal intensity & $\begin{array}{l}\text { It can depict characteristics of tissue } \\
\text { containing neuromelanin. }\end{array}$ \\
\hline $\begin{array}{l}\text { Quantitative } \\
\text { susceptibility mapping } \\
\text { (QSM) }\end{array}$ & $\begin{array}{l}\text { 3D-fast low-angle shot } \\
\text { sequence/3D fast-field } \\
\text { echo sequence }\end{array}$ & $\begin{array}{l}\text { Matlab (MathWorks, Natick, } \\
\text { MA, } \\
\text { United States)/Noxel-based } \\
\text { QSM analysis }\end{array}$ & QSM values & $\begin{array}{l}\text { It can quantify susceptibility-changing } \\
\text { materials, such as iron accumulation. }\end{array}$ \\
\hline $\begin{array}{l}\text { Diffusion tensor } \\
\text { imaging }\end{array}$ & $\begin{array}{l}\text { Pulsed gradient } \\
\text { spin-echo single- shot } \\
\text { echo-planar DT MR } \\
\text { imaging sequences }\end{array}$ & $\begin{array}{l}\text { FSL Diffusion Toolbox } \\
\text { (FMRIB Software Library, } \\
\text { Center Software Library, } \\
\text { University of Oxford, } \\
\text { Oxford, United Kingdom); }\end{array}$ & $\begin{array}{l}\text { Fractional anisotropy } \\
\text { (FA)/mean diffusivity } \\
\text { (MD) }\end{array}$ & $\begin{array}{l}\text { The directionality of movement, FA, } \\
\text { and its total magnitude, MD, both can } \\
\text { sensitively detect early microstructural } \\
\text { cortical damage. }\end{array}$ \\
\hline \multirow[t]{2}{*}{$\begin{array}{l}\text { MRI related to } \\
\text { cerebrovascular } \\
\text { abnormality }\end{array}$} & cASL/pASL & $\begin{array}{l}\text { ANTs (http: } \\
\text { //stnava.github.io/ANTs) }\end{array}$ & $\begin{array}{l}\text { Cerebral blood flow } \\
\text { (CBF) values }\end{array}$ & $\begin{array}{l}\text { Cerebrovascular abnormalities include } \\
\text { altered CBF, cerebral blood volume } \\
\text { (CBV), and blood-brain barrier } \\
\text { permeability. }\end{array}$ \\
\hline & iVASO MRI & $\begin{array}{l}\text { Matlab (MathWorks, Natick, } \\
\text { MA, United States)/SPM } \\
\text { (http: } \\
\text { //www.fil.ion.ucl.ac.uk/spm) }\end{array}$ & $\begin{array}{l}\text { Arteriolar CBV (CBVa) } \\
\text { values }\end{array}$ & $\begin{array}{l}\text { The measurement of changes in CBVa } \\
\text { may be more sensitive than } \\
\text { measurement of changes in total CBV } \\
\text { and CBF. }\end{array}$ \\
\hline $\begin{array}{l}\text { Resting-state functional } \\
\text { MRI }\end{array}$ & $\begin{array}{l}\text { Functional gradient-echo } \\
\text { EPI sequence }\end{array}$ & $\begin{array}{l}\text { SPM (http: } \\
\text { //www.fil.ion.ucl.ac.uk/spm) } \\
\text { FSL (http: } \\
\text { //www.fmrib.ox.ac.uk/fsl/) }\end{array}$ & Functional connectivity & $\begin{array}{l}\text { It can measure intrinsic blood oxygen } \\
\text { level-dependent (BOLD) low-frequency } \\
\text { signal fluctuations. }\end{array}$ \\
\hline $\begin{array}{l}\text { Proton magnetic } \\
\text { resonance } \\
\text { spectroscopy } \\
\left({ }^{1} \mathrm{H}-\mathrm{MRS}\right)\end{array}$ & PRESS sequences & $\begin{array}{l}\text { Matlab (MathWorks, Natick, } \\
\text { MA, United States)/SPM } \\
\text { (http: } \\
\text { //www.fil.ion.ucl.ac.uk/spm) }\end{array}$ & Average MRS signals & $\begin{array}{l}\text { It can reflect the integrity of different } \\
\text { elements in the brain, including } \\
N \text {-acetylaspartate (NAA), choline } \\
\text { compounds (Cho), creatine (Cr), and } \\
\text { myo-inositol (MI). }\end{array}$ \\
\hline
\end{tabular}

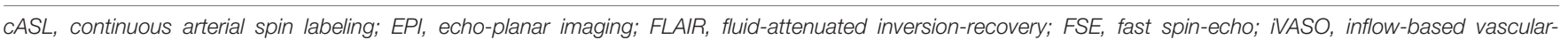

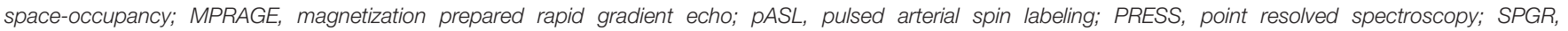
spoiled gradient echo; TFE, turbo field echo.

These typically report a pattern of linear and progressive cortical changes (reduced GM volume or cortical thinning) with increasing levels of CI (Song et al., 2011; Melzer et al., 2012; Pagonabarraga et al., 2013).

Notably, several structural MRI studies focused on assessing the differences in subcortical GM structures or ROIs crucial in the cognitive functions of PD patients. The volume of the hippocampus and substantia innominata (SI), a major source of cholinergic input to the cerebral cortex, progressively decreases corresponding to the cognitive status (Weintraub et al., 2011; Choi et al., 2012). Recent advances in the imaging technology and software analysis tools have made the study of hippocampal subregions in vivo a possibility (Iglesias et al., 2015). Smaller cornu ammonis 1 (CA1) and hippocampal-amygdaloid transition 
TABLE 2 | Summary findings from structural magnetic resonance imaging studies in Parkinson's disease and cognitive impairment.

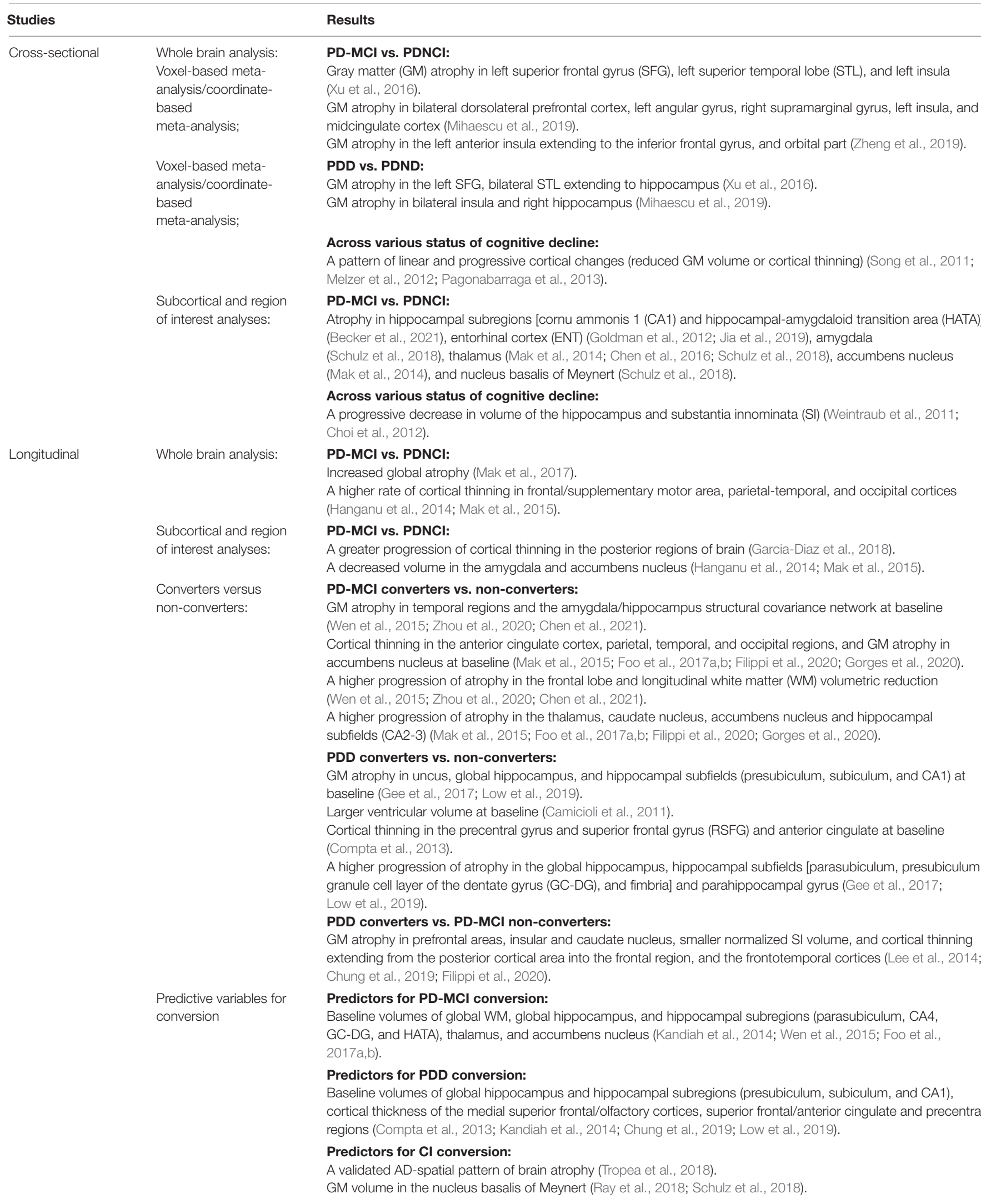

Cl, cognitive impairment; MCl, mild cognitive impairment; PD, Parkinson's disease; PDD, PD patients with dementia; PD-NCI, PD without Cl; PD-ND, PD without dementia. 
area (HATA) volumes have been observed in PD-MCI patients relative to the PD-NCI patients (Becker et al., 2021). In addition, as compared with the PD-NCI group, the PD-CI group exclusively exhibits atrophy in the entorhinal cortex (ENT) (Goldman et al., 2012; Jia et al., 2019), amygdala (Schulz et al., 2018), thalamus (Mak et al., 2014; Chen et al., 2016; Schulz et al., 2018), accumbens nucleus (Mak et al., 2014), and nucleus basalis of Meynert (Schulz et al., 2018).

\section{Longitudinal MR Studies}

Recent longitudinal studies described the progression patterns of GM changes in PD patients with CI, which can facilitate a better understanding of the mechanism of CI in PD. Patients with PD-MCI show increased global atrophy within 18 months relative to both PD-NCI patients and HCs (Mak et al., 2017). Other studies have also attempted to investigate the longitudinal changes in the cortical and subcortical GM and reported a higher rate of cortical thinning in the frontal/supplementary motor area, parietal-temporal, occipital cortices and a significant decrease in the volume of the amygdala and accumbens nucleus in PD-MCI patients relative to both PD-NCI patients and HCs (Hanganu et al., 2014; Mak et al., 2015). Some longitudinal studies have examined the differential progressive GM loss in the specific cortical regions. A 4-year follow-up study reported that over time, as compared to the PD-NCI patients, those with PD-MCI had significantly greater progression of cortical thinning in the posterior regions of the brain related to visuospatial and visuoperceptual (VS/VP) changes (Garcia-Diaz et al., 2018). Another 4-year follow-up study held the opinion that the hippocampal subfields have different vulnerabilities to the degenerative processes related to aging as well as PD. PD patients and HCs have similar longitudinal changes in CA1, while both PD-NCI and PD-MCI patients have a more severe decline in the anterior and posterior hippocampal segments, implying its predictive value for assessing memory dysfunction (Uribe et al., 2018).

\section{Converters Versus Non-converters}

Researchers have examined structural alterations in the brain to predict cognitive decline in $\mathrm{PD}$ patients. $\mathrm{PD}$ patients with normal cognition at baseline and who develop PD-MCI during follow-up are classified as "PD-MCI converters," while those who remain cognitively asymptomatic during the follow-up are classified as "non-converters." Relative to the non-converters, PD-MCI converters have a significant reduction in GM volume of temporal regions and the amygdala/hippocampus structural covariance network at baseline, along with evidently progressive frontal lobe atrophy and longitudinal white matter (WM) volumetric reduction (Wen et al., 2015; Zhou et al., 2020; Chen et al., 2021). Some studies have further investigated the pattern and progression of cortical thickness and subcortical volumes in PD-MCI converters, and reported a higher degree of anterior cingulate cortex, parietal, temporal, and occipital cortices thinning, atrophy of accumbens nucleus at baseline, and progressive atrophy in the thalamus, caudate nucleus, accumbens nucleus, and CA2-3 of hippocampal subregions
(Mak et al., 2015; Foo et al., 2017a,b; Filippi et al., 2020; Gorges et al., 2020).

Non-demented PD patients who progress to PDD during follow-up are classified as "PDD converters." At baseline, relative to the non-converters, $\mathrm{PDD}$ converters have lower GM volume in uncus, global hippocampus, and hippocampal subfields (presubiculum, subiculum, and CA1) (Gee et al., 2017; Low et al., 2019), lesser thicknesses of the precentral gyrus and superior frontal gyrus (RSFG) and anterior cingulate (Compta et al., 2013), and larger ventricular volume (Camicioli et al., 2011). Moreover, over time, PDD converters have higher GM atrophy in the global hippocampus, hippocampal subfields [parasubiculum, presubiculum, granule cell layer of the dentate gyrus (GC-DG), and fimbria] and parahippocampal gyrus (Gee et al., 2017; Low et al., 2019). Another study classified PDMCI patients as PDD converters or PD-MCI non-converters based on whether they are subsequently diagnosed with PDD during follow-up. Relative to PD-MCI non-converters, the PDD converters were found to have lower GM density in prefrontal areas, insular and caudate nucleus, smaller normalized SI volume, and a lesser cortical thickness extending from the posterior cortical area into the frontal region, and the frontotemporal cortices (Lee et al., 2014; Chung et al., 2019; Filippi et al., 2020).

\section{Predictive Variables for Conversion}

Baseline volumes of global WM, global hippocampus, and hippocampal subregions (parasubiculum, CA4, GC-DG, and HATA), thalamus, and accumbens nucleus are significant neuroimaging predictors for determining the conversion from PD-NCI to PD-MCI; the baseline global GM volume cannot significantly predict the conversion to PD-MCI (Kandiah et al., 2014; Wen et al., 2015; Foo et al., 2017a,b). Moreover, GM volume in the global hippocampus and hippocampal subregions (presubiculum, subiculum, and CA1), cortical thickness in the medial superior frontal/olfactory cortices, superior frontal/anterior cingulate and precentral regions are significant predictors for the progression to PDD (Compta et al., 2013; Kandiah et al., 2014; Chung et al., 2019; Low et al., 2019).

Growing evidence highlighted that brain regions undergoing neurodegeneration may overlap synergistically with the pathological processes for PD and Alzheimer's disease (AD) (Masliah et al., 2001; Clinton et al., 2010). To determine its association with $\mathrm{CI}$, a validated $\mathrm{AD}$-spatial pattern of brain atrophy (spatial pattern of abnormality for recognition of early $\mathrm{AD}$ index, SPARE-AD) was quantified in PD patients with a range of cognitive abilities. The findings support the involvement of hippocampus and parietal-temporal cortex with CI in PD (Weintraub et al., 2012), and affirm the importance of $\mathrm{AD}$ biomarkers as effective predictors for CI in PD (Tropea et al., 2018). In addition, autopsies have identified degeneration of the cholinergic basal forebrain, especially in the nucleus basalis of Meynert, causing cortical cholinergic depletion, and eventually leading to the CI in patients with PD and AD (Liu et al., 2015). GM volume in the nucleus basalis of Meynert was a critical cholinergic biomarker to predict CI in PD-NCI patients (Ray et al., 2018; Schulz et al., 2018). 


\section{OTHER MAGNETIC RESONANCE IMAGING TECHNIQUES}

\section{Magnetic Resonance Imaging Related to Small-Vessel Disease}

As a consequence of cerebral small-vessel disease (SVD), white matter hyperintensities (WMHs) in the brain can reflect pathological WM tissue ischemia (Erten-Lyons et al., 2013). PD patients with greater WMH have more severe cognitive deficits and a higher annual rate of change in global cognition (Dadar et al., 2018; Chahine et al., 2019; Chang et al., 2021). The periventricular WMH burden is significantly associated with $\mathrm{PD}$ MCI and severe WMH with PDD; both are independent of cardiovascular risk factors (Huang et al., 2020; Tsai et al., 2021). In addition, heavier temporal WMH burden is associated with worse verbal memory (Chahine et al., 2019), and higher periventricular WMH burden correlates with greater decline in the executive and visuospatial functions (Huang et al., 2020). Therefore, WMH is strongly correlated with $\mathrm{CI}$ in PD and may serve as a surrogate marker for CI in PD (Kandiah et al., 2014; Fang et al., 2021). Visible perivascular space (PVS) has also been considered as an imaging biomarker for SVD. A longitudinal MRI-visible PVS study reported higher PVS severity in basal ganglia (BG) as a predictor of cognitive conversion of PD-NCI patients as well as for cognitive decline in PD-MCI patients (Park et al., 2019). Summary findings from other MRI techniques correlated with cognitive dysfunction in PD can be found in Table 3.

\section{Neuromelanin-Sensitive Magnetic Resonance Imaging}

Neuromelanin-sensitive MRI (NM-MRI) is a promising technique that depicts characteristics of tissue containing neuromelanin, a dark polymer product of noradrenaline metabolism (Nakamura and Sugaya, 2014). The substantia nigra (SN) of the midbrain and the locus coeruleus (LC) within the pons contain large clusters of neuromelanin-producing cells, and the loss of these neuromelanin-containing cells within the two regions is a primary pathological diagnostic criterion for PD. The NM signal intensity of the LC in PD-MCI patients is significantly lower than that of the control subjects, and may serve as an imaging marker for assessing cognitive dysfunction in PD (Li et al., 2019; Prasuhn et al., 2021).

\section{Quantitative Susceptibility Mapping}

Quantitative susceptibility mapping (QSM) is another new MRI technique that enables the quantification of susceptibilitychanging materials. Iron accumulation has been proposed as a significant pathomechanism for PD, which can be systematically examined using the QSM method. PD-MCI patients have significantly higher QSM values in the orbitofrontal cortex, cuneus, precuneus, fusiform gyrus, and head of the caudate nucleus relative to the PD-NCI patients (Uchida et al., 2019). Meanwhile, PDD patients have significantly higher QSM values in the left hippocampus as compared to non-demented PD patients (Li et al., 2018). These findings suggest the association between $\mathrm{CI}$ in PD and cerebral iron burden, which may be an auxiliary biomarker for early detection of CI in PD.

\section{MR Diffusion Imaging}

Diffusion tensor imaging (DTI) is an in vivo surrogate measure for the microstructural integrity of WM tracts. Using this technique, the rate of 3-dimensional water molecule diffusion through a pulsed-field gradient can be measured. The directionality of movement is quantified as fractional anisotropy (FA) and its total magnitude is measured as mean diffusivity (MD). The decrease in FA and increase in $\mathrm{MD}$ within the central nervous system have been proposed as sensitive imaging indicators for detecting early microstructural cortical damage in patients with neurodegenerative disorders.

The PD-MCI patients have a distributed pattern of WM abnormalities in the anterior and superior corona radiata, genu, and body of the corpus callosum, anterior inferior fronto-occipital, uncinate, and superior longitudinal fasciculi relative to PD-NCI patients and HCs (Agosta et al., 2014). Lower FA values in frontotemporoparietal regions, hippocampus, amygdala, thalamus, BG, and higher MD values in hippocampus, ENT, amygdala, thalamus, and nucleus basalis of Meynert relative to PD-NCI patients have been reported in PD-MCI patients (Galantucci et al., 2017; Schulz et al., 2018). PDD patients have significantly lower FA values in the prefrontal WM, hippocampus, and the genu of the corpus callosum relative to the non-demented PD patients (Kamagata et al., 2013; Chen et al., 2015), and lower FA values in both the anterior and the posterior cingulate fiber tracts as compared to HCs (Kamagata et al., 2012). Microstructural abnormalities in WM of PD patients with variant cognitive statuses (PD-NCI, PD-MCI, and PDD) have been investigated to elucidate the pathological processes of $\mathrm{CI}$ in PD. The loss of microstructural WM integrity is spatially restricted in PD-NCI patients, while these alterations increase with progressive cognitive deterioration (Hattori et al., 2012; Deng et al., 2013; Melzer et al., 2013).

In a previous study, dynamic MD changes were specifically assessed by a longitudinal approach in newly-diagnosed drugnaïve PD patients. A higher 1-year MD increase in frontal and occipital cortices was found in PD patients relative to $\mathrm{HCs}$, which correlated with changes in the cognitive measures (Sampedro et al., 2019). Moreover, the increased MD values in the nucleus basalis of Meynert can predict the conversion from PD-NCI to PD with CI (Schulz et al., 2018). Thus, further longitudinal investigations are required to establish the clinical utility of DTI as a sensitive biomarker for the pathology of CI in PD.

\section{Magnetic Resonance Imaging Related to Cerebrovascular Abnormality}

Emerging evidence supported that cerebrovascular abnormalities including altered cerebral blood flow (CBF), cerebral blood volume $(\mathrm{CBV})$, and blood-brain barrier permeability are linked with pathophysiology in CI. Using the arterial spin labeling (ASL) technique, brain perfusion can be quantitatively assessed in terms of CBF. A pattern of decreased CBF at the posterior part of the brain, mostly in the lateral and medial parietooccipital cortices, 
TABLE 3 | Summary findings from other magnetic resonance imaging studies in Parkinson's disease and cognitive impairment.

\begin{tabular}{|c|c|c|}
\hline Modalities & Results & \\
\hline \multirow[t]{4}{*}{$\begin{array}{l}\text { MRI related to } \\
\text { small-vessel disease }\end{array}$} & \multirow{3}{*}{$\begin{array}{l}\text { White matter } \\
\text { hyperintensity } \\
(\text { WMH) }\end{array}$} & $\begin{array}{l}\text { PD patients with greater WMH have more severe cognitive deficits and a higher annual rate of change in global } \\
\text { cognition (Dadar et al., 2018; Chahine et al., 2019; Chang et al., 2021). }\end{array}$ \\
\hline & & $\begin{array}{l}\text { The periventricular WMH burden is significantly associated with PD-MCl and severe WMH with PDD (Huang et al., } \\
\text { 2020; Tsai et al., 2021). }\end{array}$ \\
\hline & & $\begin{array}{l}\text { WMH is strongly correlated with } \mathrm{Cl} \text { in PD and may serve as a surrogate marker for Cl in PD (Kandiah et al., 2014; } \\
\text { Fang et al., 2021). }\end{array}$ \\
\hline & $\begin{array}{l}\text { Perivascular space } \\
\text { (PVS) }\end{array}$ & $\begin{array}{l}\text { Higher PVS severity in basal ganglia (BG) as a predictor of cognitive conversion of PD-NCI patients as well as for } \\
\text { cognitive decline in PD-MCl patients (Park et al., 2019). }\end{array}$ \\
\hline Neuromelanin-sensitive & \multirow[t]{2}{*}{ Signal intensity } & PD-MCl vs. HC \\
\hline MRI (NM-MRI) & & $\begin{array}{l}\text { Lower NM signal intensity in the locus coeruleus, which may serve as an imaging marker for assessing cognitive } \\
\text { dysfunction in PD (Li et al., 2019; Prasuhn et al., 2021). }\end{array}$ \\
\hline Quantitative & \multirow[t]{4}{*}{ QSM values } & PD-MCI vs. PDNCI \\
\hline \multirow[t]{3}{*}{$\begin{array}{l}\text { susceptibility mapping } \\
\text { (QSM) }\end{array}$} & & $\begin{array}{l}\text { Higher QSM values in the orbitofrontal cortex, cuneus, precuneus, fusiform gyrus, and head of the caudate } \\
\text { nucleus (Uchida et al., 2019). }\end{array}$ \\
\hline & & PDD vs. PDND \\
\hline & & Higher QSM values in the left hippocampus (Li et al., 2018). \\
\hline \multirow{8}{*}{$\begin{array}{l}\text { Diffusion tensor } \\
\text { imaging }\end{array}$} & \multirow{8}{*}{$\begin{array}{l}\text { Fractional } \\
\text { anisotropy } \\
\text { (FA)/mean diffusivity } \\
\text { (MD) }\end{array}$} & PD-MCI vs. PDNCI \\
\hline & & $\begin{array}{l}\text { Decreased FA values in the anterior and superior corona radiata, genu, and body of the corpus callosum, anterior } \\
\text { inferior fronto-occipital fasciculus (IFOF)/uncinate, and anterior superior longitudinal fasciculi (SLF), } \\
\text { frontotemporoparietal regions, hippocampus, BG, amygdala, thalamus (Agosta et al., 2014; Galantucci et al., } \\
\text { 2017; Schulz et al., 2018). } \\
\text { Increased MD values in hippocampus, entorhinal cortex (ENT), amygdala, thalamus, and nucleus basalis of } \\
\text { Meynert (Galantucci et al., 2017; Schulz et al., 2018). }\end{array}$ \\
\hline & & PDD vs. PDND \\
\hline & & $\begin{array}{l}\text { Decreased FA values in the prefrontal white matter, hippocampus, and the genu of the corpus callosum } \\
\text { (Kamagata et al., 2013; Chen et al., 2015). }\end{array}$ \\
\hline & & Across various status of cognitive decline \\
\hline & & $\begin{array}{l}\text { The loss of microstructural WM integrity was spatially restricted in PD-NCl patients, while these alterations } \\
\text { increase with progressive cognitive deterioration (Hattori et al., 2012; Deng et al., 2013; Melzer et al., 2013). }\end{array}$ \\
\hline & & Predictors for $\mathrm{Cl}$ conversion \\
\hline & & The increased MD values in the nucleus basalis of Meynert (Schulz et al., 2018). \\
\hline \multirow[t]{3}{*}{$\begin{array}{l}\text { MRI related to } \\
\text { cerebrovascular } \\
\text { abnormality }\end{array}$} & $\begin{array}{l}\text { Cerebral blood flow } \\
\text { (CBF) values }\end{array}$ & $\begin{array}{l}\text { PD-MCI vs. HC } \\
\text { A pattern of decreased CBF at the posterior part of the brain, mostly in the lateral and medial parietooccipital } \\
\text { cortices, summarized as "posterior hypoperfusion" (Arslan et al., 2020). } \\
\text { Parietal CBF may serve as a potential early biomarker for Cl in PD (Pelizzari et al., 2020). }\end{array}$ \\
\hline & \multirow{2}{*}{$\begin{array}{l}\text { Arteriolar cerebral } \\
\text { blood volume } \\
\text { (CBVa) values }\end{array}$} & PD-Cl vs. HC \\
\hline & & $\begin{array}{l}\text { A decreasing trend of CBVa in the substantia nigra, caudate nucleus and putamen, and an increasing trend of } \\
\text { CBVa in the pre-supplementary motor area and intracalcarine gyrus (Paez et al., 2020). }\end{array}$ \\
\hline \multirow{4}{*}{$\begin{array}{l}\text { Resting-state functional } \\
\text { MRI }\end{array}$} & \multirow{4}{*}{$\begin{array}{l}\text { Functional } \\
\text { connectivity (FC) }\end{array}$} & PD-Cl vs. PDNCI \\
\hline & & Reduced FC in specific brain regions that are parts of the default mode network (DMN) (Wolters et al., 2019). \\
\hline & & Predictors for $\mathrm{Cl}$ conversion \\
\hline & & $\begin{array}{l}\text { FC between the medial prefrontal cortex (mPFC) and posterior cingulate cortex (PCC) within DMN at baseline } \\
\text { (Zarifkar et al., 2021). }\end{array}$ \\
\hline \multirow[t]{2}{*}{$\begin{array}{l}\text { Proton magnetic } \\
\text { resonance } \\
\text { spectroscopy } \\
\left({ }^{1} \mathrm{H}-\mathrm{MRS}\right)\end{array}$} & \multirow[t]{2}{*}{$\begin{array}{l}\text { Average MRS } \\
\text { signals }\end{array}$} & $\begin{array}{l}\text { PD-CI vs. PDNCI } \\
\text { Higher the choline to creatine (Cho/Cr) ratio in the occipital lobe, reduced concentration of } N \text {-acetylaspartate } \\
\text { (NAA) in the middle and superior frontal gray matter regions, and reduced concentration of total Cho and total } \mathrm{Cr} \\
\text { in the frontal white matter regions (Nie et al., 2013; Chaudhary et al., 2021). }\end{array}$ \\
\hline & & $\begin{array}{l}\text { Levels of NAA reduced in the dorsolateral prefrontal cortex in the early stage, and progression to the } \\
\text { hippocampus in PDD patients (Pagonabarraga et al., 2012). } \\
\text { Frontal neuro-metabolism may serve as a promising biomarker for characterizing cognitive deficits in patients with } \\
\text { PD (Chaudhary et al., 2021). }\end{array}$ \\
\hline
\end{tabular}

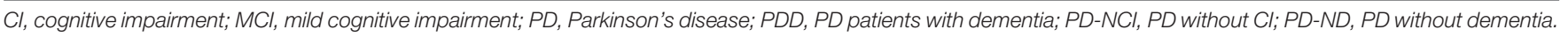

summarized as "posterior hypoperfusion," has been reported in the PD-MCI group relative to HCs (Arslan et al., 2020), which may be a suitable early biomarker for CI in PD. Parietal CBF correlates to visuomotor skills and also may serve as a potential early biomarker for CI in PD (Pelizzari et al., 2020). Using the inflow-based vascular-space-occupancy (iVASO) MRI approach, potential arteriolar CBV (CBVa) abnormalities reflecting the homeostasis of the most actively regulated segment in the microvasculature can be calculated. A decreasing trend of CBVa in the substantia nigra, caudate nucleus and putamen, and an increasing trend of CBVa in the pre-supplementary motor area and intracalcarine gyrus have been reported in PD-CI patients 
relative to HCs (Paez et al., 2020). These exploratory findings provide important information for efforts toward developing potential biomarkers for the risk of $\mathrm{CI}$ in $\mathrm{PD}$.

\section{Resting-State Functional Magnetic Resonance Imaging}

Resting-state functional MRI (rs-fMRI) can provide a reliable and reproducible tool to examine the functional activity in different brain networks by measuring intrinsic blood oxygen leveldependent (BOLD) low-frequency signal fluctuations. There is a growing interest in using fMRI to investigate the neural basis for CI in PD.

A voxel-based meta-analysis consisting of 289 PD-NCI, $222 \mathrm{PD}-\mathrm{MCI}, 68 \mathrm{PDD}$, and $353 \mathrm{HC}$ subjects from seventeen studies showed that $\mathrm{PD}$ patients with $\mathrm{CI}$ had reduced functional connectivity (FC) in specific brain regions that are parts of the default mode network (DMN) (Wolters et al., 2019). Moreover, notably, increased FC may be the initial manifestation of altered brain function preceding cognitive deficits (transition from PD-NCI to PD-MCI), and these functional disruptions may be considerably associated with a cognitive decline in the progression of PD-related pathology (Gorges et al., 2015; Zhan et al., 2018; Zarifkar et al., 2021). A novel graphtheoretical analysis of functional networks indicates that the topological properties of brain networks are disrupted in PDMCI patients with reduced long-range connections and enhanced local interconnectedness (Baggio et al., 2014). Moreover, a recent study suggested that $\mathrm{PD}-\mathrm{MCI}$ patients had an increased random organization of brain networks and altered nodal centralities as compared with HCs (Hou et al., 2020). These results provide a new perspective on the functional basis of $\mathrm{CI}$ in $\mathrm{PD}$.

In an exploratory longitudinal study, FC between the medial prefrontal cortex (mPFC) and posterior cingulate cortex (PCC) within DMN at baseline were found to be the best putative predictor for CI in PD (Zarifkar et al., 2021). PD patients with a longitudinal decline in cognitive function have a significant decline in dorsal attention-frontoparietal internetwork (DAN-FPN) strength, which may serve as a prognostic marker for assessing the development of dementia in PD (Campbell et al., 2020).

\section{Proton Magnetic Resonance Spectroscopy}

Proton magnetic resonance spectroscopy ( $\left.{ }^{1} \mathrm{H}-\mathrm{MRS}\right)$ owing to a high natural abundance and magnetic sensitivity of protons in the brain allows for in vivo measurements of four major metabolites, that reflect the integrity of different elements in the

\section{REFERENCES}

Aarsland, D., and Kurz, M. W. (2010). The epidemiology of dementia associated with Parkinson disease. J. Neurol. Sci. 289, 18-22. doi: 10.1016/j.jns.2009.08.034

Agosta, F., Canu, E., Stefanova, E., Sarro, L., Tomic, A., Spica, V., et al. (2014). Mild cognitive impairment in Parkinson's disease is associated with a distributed brain, including $N$-acetylaspartate (NAA), choline compounds (Cho), creatine (Cr), and myo-inositol (MI) (Nie et al., 2013).

The $\mathrm{PD}-\mathrm{CI}$ patients have higher the choline to creatine $(\mathrm{Cho} / \mathrm{Cr})$ ratio in the occipital lobe, reduced concentration of NAA in the middle and superior frontal gray matter regions, and reduced concentration of total $\mathrm{Cho}$ and total $\mathrm{Cr}$ in the frontal white matter regions relative to $\mathrm{PD}-\mathrm{CN}$ patients and HCs (Nie et al., 2013; Chaudhary et al., 2021). According to the differential status of CI, levels of NAA in the dorsolateral prefrontal cortex are reduced in the early stage with progression to the hippocampus in PDD patients (Pagonabarraga et al., 2012). Frontal neuro-metabolism may serve as a promising biomarker for characterizing cognitive deficits in patients with PD (Chaudhary et al., 2021).

\section{CONCLUSION}

In summary, specific patterns of cognitive neural substrates in PD can be detected using various MRI techniques, and this has become a putative prediction tool for CI in PD. With the advances in image acquisition and analysis technologies, predictors derived from structural MRI are promising for assessing the cognitive dysfunction in PD. Recently, abnormal patterns from other MRI techniques also have emerged as the candidate biomarkers for evaluating the progression of cognitive decline in PD. Given that biomarkers from a single modal MRI cannot completely describe the complexity of pathological substrates of cognitive deficits in $\mathrm{PD}$, we believe that the potential predictors from multi-modal MRI technology using recognized analysis tools and predictive algorithms deserve further investigation. Furthermore, targeted biomarkers for different cognitive domains and the determination of the best combination scheme of biomarkers from multi-modal MRI are the most important issues in the future.

\section{AUTHOR CONTRIBUTIONS}

YH prepared the initial manuscript. HS provided content expertise and reviewed the manuscript. Both authors contributed to the article and approved the submitted version.

\section{FUNDING}

This present study was supported by the Post-doctor Research Project, West China Hospital, Sichuan University (2020HXBH100 to YH).

pattern of brain white matter damage. Hum. Brain Mapp. 35, 1921-1929. doi: 10.1002/hbm.22302

Arslan, D. B., Gurvit, H., Genc, O., Kicik, A., Eryurek, K., Cengiz, S., et al. (2020). The cerebral blood flow deficits in Parkinson's disease with mild cognitive impairment using arterial spin labeling MRI. J. Neural Transm. (Vienna) 127, 1285-1294. doi: 10.1007/s00702-020-02227-6 
Baggio, H. C., Sala-Llonch, R., Segura, B., Marti, M. J., Valldeoriola, F., Compta, Y., et al. (2014). Functional brain networks and cognitive deficits in Parkinson's disease. Hum. Brain Mapp. 35, 4620-4634. doi: 10.1002/hbm.22499

Baiano, C., Barone, P., Trojano, L., and Santangelo, G. (2020). Prevalence and clinical aspects of mild cognitive impairment in Parkinson's disease: a metaanalysis. Mov. Disord. 35, 45-54. doi: 10.1002/mds.27902

Becker, S., Granert, O., Timmers, M., Pilotto, A., Van Nueten, L., Roeben, B., et al. (2021). Association of hippocampal subfields, CSF biomarkers, and cognition in patients with parkinson disease without dementia. Neurology 96, e904-e915. doi: 10.1212/wnl.0000000000011224

Bloem, B. R., Okun, M. S., and Klein, C. (2021). Parkinson's disease. Lancet 397, 2284-2303. doi: 10.1016/S0140-6736(21)00218-X

Bohnen, N. I., and Albin, R. L. (2011). White matter lesions in Parkinson disease. Nat. Rev. Neurol. 7, 229-236. doi: 10.1038/nrneurol.2011.21

Broeders, M., de Bie, R. M., Velseboer, D. C., Speelman, J. D., Muslimovic, D., and Schmand, B. (2013). Evolution of mild cognitive impairment in Parkinson disease. Neurology 81, 346-352. doi: 10.1212/WNL.0b013e31829c5c86

Camicioli, R., Sabino, J., Gee, M., Bouchard, T., Fisher, N., Hanstock, C., et al. (2011). Ventricular dilatation and brain atrophy in patients with Parkinson's disease with incipient dementia. Mov. Disord. 26, 1443-1450. doi: 10.1002/mds. 23700

Campbell, M. C., Jackson, J. J., Koller, J. M., Snyder, A. Z., Kotzbauer, P. T., and Perlmutter, J. S. (2020). Proteinopathy and longitudinal changes in functional connectivity networks in Parkinson disease. Neurology 94, e718-e728. doi: 10. 1212/wnl.0000000000008677

Chahine, L. M., Dos Santos, C., Fullard, M., Scordia, C., Weintraub, D., Erus, G., et al. (2019). Modifiable vascular risk factors, white matter disease and cognition in early Parkinson's disease. Eur. J. Neurol. 26, 246-e218. doi: 10.1111/ene. 13797

Chang, T. C., Chen, Y. C., Huang, Y. C., Lin, W. C., and Lu, C. H. (2021). Systemic oxidative stress and cognitive function in Parkinson's disease with different PWMH or DWMH lesions. BMC Neurol. 21:16. doi: 10.1186/s12883020-02037-z

Chaudhary, S., Kumaran, S. S., Goyal, V., Kalaivani, M., Kaloiya, G. S., Sagar, R., et al. (2021). Frontal lobe metabolic alterations characterizing Parkinson's disease cognitive impairment. Neurol. Sci. 42, 1053-1064. doi: 10.1007/s10072020-04626-9

Chen, B. Y., Fan, G. G., Liu, H., and Wang, S. S. (2015). Changes in anatomical and functional connectivity of Parkinson's disease patients according to cognitive status. Eur. J. Radiol. 84, 1318-1324. doi: 10.1016/j.ejrad.2015.04.014

Chen, F. X., Kang, D. Z., Chen, F. Y., Liu, Y., Wu, G., Li, X., et al. (2016). Gray matter atrophy associated with mild cognitive impairment in Parkinson's disease. Neurosci. Lett. 617, 160-165. doi: 10.1016/j.neulet.2015. 12.055

Chen, Y. S., Chen, H. L., Lu, C. H., Lee, C. Y., Chou, K. H., Chen, M. H., et al. (2021). The corticolimbic structural covariance network as an early predictive biosignature for cognitive impairment in Parkinson's disease. Sci. Rep. 11:862. doi: 10.1038/s41598-020-79403-x

Choi, S. H., Jung, T. M., Lee, J. E., Lee, S. K., Sohn, Y. H., and Lee, P. H. (2012). Volumetric analysis of the substantia innominata in patients with Parkinson's disease according to cognitive status. Neurobiol. Aging 33, 1265-1272. doi: 10.1016/j.neurobiolaging.2010.11.015

Chung, S. J., Yoo, H. S., Lee, Y. H., Lee, H. S., Ye, B. S., Sohn, Y. H., et al. (2019). Frontal atrophy as a marker for dementia conversion in Parkinson's disease with mild cognitive impairment. Hum. Brain Mapp. 40, 3784-3794. doi: $10.1002 / \mathrm{hbm} .24631$

Clinton, L. K., Blurton-Jones, M., Myczek, K., Trojanowski, J. Q., and LaFerla, F. M. (2010). Synergistic Interactions between Abeta, tau, and alpha-synuclein: acceleration of neuropathology and cognitive decline. J. Neurosci. 30, 72817289. doi: 10.1523/JNEUROSCI.0490-10.2010

Collaborators, G. B. D. N. (2019). Global, regional, and national burden of neurological disorders, 1990-2016: a systematic analysis for the global burden of disease study 2016. Lancet Neurol. 18, 459-480. doi: 10.1016/S1474-4422(18) 30499-X

Compta, Y., Pereira, J. B., Ríos, J., Ibarretxe-Bilbao, N., Junqué, C., Bargalló, N., et al. (2013). Combined dementia-risk biomarkers in Parkinson's disease: a prospective longitudinal study. Parkinsonism Relat. Disord. 19, 717-724. doi: 10.1016/j.parkreldis.2013.03.009
Dadar, M., Zeighami, Y., Yau, Y., Fereshtehnejad, S. M., Maranzano, J., Postuma, R. B., et al. (2018). White matter hyperintensities are linked to future cognitive decline in de novo Parkinson's disease patients. Neuroimage Clin. 20, 892-900. doi: 10.1016/j.nicl.2018.09.025

de Boer, R., Vrooman, H. A., van der Lijn, F., Vernooij, M. W., Ikram, M. A., van der Lugt, A., et al. (2009). White matter lesion extension to automatic brain tissue segmentation on MRI. Neuroimage 45, 1151-1161. doi: 10.1016/ j.neuroimage.2009.01.011

Deng, B. M., Zhang, Y. H., Wang, L. J., Peng, K. R., Han, L. X., Nie, K., et al. (2013). Diffusion tensor imaging reveals white matter changes associated with cognitive status in patients with Parkinson's disease. Am. J. Alzheimers Dis. Other Dement. 28, 154-164. doi: 10.1177/1533317512470207

Dorsey, E. R., Sherer, T., Okun, M. S., and Bloem, B. R. (2018). The emerging evidence of the Parkinson pandemic. J. Parkinsons Dis. 8, S3-S8. doi: 10.3233/ JPD- 181474

Emre, M., Aarsland, D., Brown, R., Burn, D. J., Duyckaerts, C., Mizuno, Y., et al. (2007). Clinical diagnostic criteria for dementia associated with Parkinson's disease. Mov. Disord. 22, 1689-1707; quiz1837. doi: 10.1002/mds.21507

Erten-Lyons, D., Woltjer, R., Kaye, J., Mattek, N., Dodge, H. H., Green, S., et al. (2013). Neuropathologic basis of white matter hyperintensity accumulation with advanced age. Neurology 81, 977-983. doi: 10.1212/WNL. 0b013e3182a43e45

Fang, E., Fartaria, M. J., Ann, C. N., Maréchal, B., Kober, T., Lim, J. X., et al. (2021). Clinical correlates of white matter lesions in Parkinson's disease using automated multi-modal segmentation measures. J. Neurol. Sci. 427:117518. doi: 10.1016/j.jns.2021.117518

Filippi, M., Canu, E., Donzuso, G., Stojkovic, T., Basaia, S., Stankovic, I., et al. (2020). Tracking cortical changes throughout cognitive decline in Parkinson's disease. Mov. Disord. 35, 1987-1998. doi: 10.1002/mds.28228

Foo, H., Mak, E., Chander, R. J., Ng, A., Au, W. L., Sitoh, Y. Y., et al. (2017a). Associations of hippocampal subfields in the progression of cognitive decline related to Parkinson's disease. Neuroimage Clin. 14, 37-42. doi: 10.1016/j.nicl. 2016.12.008

Foo, H., Mak, E., Yong, T. T., Wen, M. C., Chander, R. J., Au, W. L., et al. (2017b). Progression of subcortical atrophy in mild Parkinson's disease and its impact on cognition. Eur. J. Neurol. 24, 341-348. doi: 10.1111/ene.13205

Galantucci, S., Agosta, F., Stefanova, E., Basaia, S., van den Heuvel, M. P., Stojković, T., et al. (2017). Structural brain connectome and cognitive impairment in Parkinson disease. Radiology 283, 515-525. doi: 10.1148/radiol.2016160274

Garcia-Diaz, A. I., Segura, B., Baggio, H. C., Uribe, C., Campabadal, A., Abos, A., et al. (2018). Cortical thinning correlates of changes in visuospatial and visuoperceptual performance in Parkinson's disease: a 4-year follow-up. Parkinsonism Relat. Disord. 46, 62-68. doi: 10.1016/j.parkreldis.2017.11.003

Gee, M., Dukart, J., Draganski, B., Martin, W. R. W., Emery, D., and Camicioli, R. (2017). Regional volumetric change in Parkinson's disease with cognitive decline. J. Neurol. Sci. 373, 88-94. doi: 10.1016/j.jns.2016.12.030

Goldman, J. G., Stebbins, G. T., Bernard, B., Stoub, T. R., Goetz, C. G., and deToledo-Morrell, L. (2012). Entorhinal cortex atrophy differentiates Parkinson's disease patients with and without dementia. Mov. Disord. 27, 727-734. doi: 10.1002/mds.24938

Gorges, M., Kunz, M. S., Muller, H. P., Liepelt-Scarfone, I., Storch, A., Dodel, R., et al. (2020). Longitudinal brain atrophy distribution in advanced Parkinson's disease: what makes the difference in "cognitive status" converters? Hum. Brain Mapp. 41, 1416-1434. doi: 10.1002/hbm.24884

Gorges, M., Muller, H. P., Lule, D., Consortium, L., Pinkhardt, E. H., Ludolph, A. C., et al. (2015). To rise and to fall: functional connectivity in cognitively normal and cognitively impaired patients with Parkinson's disease. Neurobiol. Aging 36, 1727-1735. doi: 10.1016/j.neurobiolaging.2014.12.026

Hanganu, A., Bedetti, C., Degroot, C., Mejia-Constain, B., Lafontaine, A.-L., Soland, V., et al. (2014). Mild cognitive impairment is linked with faster rate of cortical thinning in patients with Parkinson's disease longitudinally. Brain 137, 1120-1129. doi: 10.1093/brain/awu036

Hattori, T., Orimo, S., Aoki, S., Ito, K., Abe, O., Amano, A., et al. (2012). Cognitive status correlates with white matter alteration in Parkinson's disease. Hum. Brain Mapp. 33, 727-739. doi: 10.1002/hbm.21245

Hou, Y., Wei, Q., Ou, R., Yang, J., Gong, Q., and Shang, H. (2020). Impaired topographic organization in Parkinson's disease with mild cognitive impairment. J. Neurol. Sci. 414, 116861. doi: 10.1016/j.jns.2020.116861 
Huang, X., Wen, M. C., Ng, S. Y., Hartono, S., Chia, N. S., Choi, X., et al. (2020). Periventricular white matter hyperintensity burden and cognitive impairment in early Parkinson's disease. Eur. J. Neurol. 27, 959-966. doi: 10.1111/ene. 14192

Iglesias, J. E., Augustinack, J. C., Nguyen, K., Player, C. M., Player, A., Wright, M., et al. (2015). A computational atlas of the hippocampal formation using ex vivo, ultra-high resolution MRI: application to adaptive segmentation of in vivo MRI. Neuroimage 115, 117-137. doi: 10.1016/j.neuroimage.2015.04.042

Jia, X., Wang, Z., Yang, T., Li, Y., Gao, S., Wu, G., et al. (2019). Entorhinal cortex atrophy in early, drug-naive Parkinson's disease with mild cognitive impairment. Aging Dis. 10, 1221-1232. doi: 10.14336/ad.2018.1116

Kamagata, K., Motoi, Y., Abe, O., Shimoji, K., Hori, M., Nakanishi, A., et al. (2012). White matter alteration of the cingulum in Parkinson disease with and without dementia: evaluation by diffusion tensor tract-specific analysis. Am. J. Neuroradiol. 33, 890-895. doi: 10.3174/ajnr.A2860

Kamagata, K., Motoi, Y., Tomiyama, H., Abe, O., Ito, K., Shimoji, K., et al. (2013). Relationship between cognitive impairment and white-matter alteration in Parkinson's disease with dementia: tract-based spatial statistics and tract-specific analysis. Eur. Radiol. 23, 1946-1955. doi: 10.1007/s00330-0132775-4

Kandiah, N., Zainal, N. H., Narasimhalu, K., Chander, R. J., Ng, A., Mak, E., et al. (2014). Hippocampal volume and white matter disease in the prediction of dementia in Parkinson's disease. Parkinsonism Relat. Disord. 20, 1203-1208. doi: 10.1016/j.parkreldis.2014.08.024

Lee, J. E., Cho, K. H., Song, S. K., Kim, H. J., Lee, H. S., Sohn, Y. H., et al. (2014). Exploratory analysis of neuropsychological and neuroanatomical correlates of progressive mild cognitive impairment in Parkinson's disease. J. Neurol. Neurosurg. Psychiatry 85, 7-16. doi: 10.1136/jnnp-2013-305062

Li, D. T. H., Hui, E. S., Chan, Q., Yao, N., Chua, S. E., McAlonan, G. M., et al. (2018). Quantitative susceptibility mapping as an indicator of subcortical and limbic iron abnormality in Parkinson's disease with dementia. Neuroimage Clin. 20, 365-373. doi: 10.1016/j.nicl.2018.07.028

Li, Y., Wang, C., Wang, J., Zhou, Y., Ye, F., Zhang, Y., et al. (2019). Mild cognitive impairment in de novo Parkinson's disease: a neuromelanin MRI study in locus coeruleus. Mov. Disord. 34, 884-892. doi: 10.1002/mds.27682

Litvan, I., Goldman, J. G., Troster, A. I., Schmand, B. A., Weintraub, D., Petersen, R. C., et al. (2012). Diagnostic criteria for mild cognitive impairment in Parkinson's disease: movement disorder society task force guidelines. Mov. Disord. 27, 349-356. doi: 10.1002/mds.24893

Liu, A. K., Chang, R. C., Pearce, R. K., and Gentleman, S. M. (2015). Nucleus basalis of Meynert revisited: anatomy, history and differential involvement in Alzheimer's and Parkinson's disease. Acta Neuropathol. 129, 527-540. doi: 10.1007/s00401-015-1392-5

Low, A., Foo, H., Yong, T. T., Tan, L. C. S., and Kandiah, N. (2019). Hippocampal subfield atrophy of CA1 and subicular structures predict progression to dementia in idiopathic Parkinson's disease. J. Neurol. Neurosurg. Psychiatry 90, 681-687. doi: 10.1136/jnnp-2018-319592

Mak, E., Bergsland, N., Dwyer, M. G., Zivadinov, R., and Kandiah, N. (2014). Subcortical atrophy is associated with cognitive impairment in mild Parkinson disease: a combined investigation of volumetric changes, cortical thickness, and vertex-based shape analysis. AJNR Am. J. Neuroradiol. 35, 2257-2264. doi: 10.3174/ajnr.A4055

Mak, E., Su, L., Williams, G. B., Firbank, M. J., Lawson, R. A., Yarnall, A. J., et al. (2015). Baseline and longitudinal grey matter changes in newly diagnosed Parkinson's disease: ICICLE-PD study. Brain 138, 2974-2986. doi: 10.1093/ brain/awv211

Mak, E., Su, L., Williams, G. B., Firbank, M. J., Lawson, R. A., Yarnall, A. J., et al. (2017). Longitudinal whole-brain atrophy and ventricular enlargement in nondemented Parkinson's disease. Neurobiol. Aging 55, 78-90. doi: 10.1016/j. neurobiolaging.2017.03.012

Masliah, E., Rockenstein, E., Veinbergs, I., Sagara, Y., Mallory, M., Hashimoto, M., et al. (2001). beta-amyloid peptides enhance alpha-synuclein accumulation and neuronal deficits in a transgenic mouse model linking Alzheimer's disease and Parkinson's disease. Proc. Natl. Acad. Sci. U.S.A. 98, 12245-12250. doi: 10.1073/pnas. 211412398

Melzer, T. R., Watts, R., MacAskill, M. R., Pitcher, T. L., Livingston, L., Keenan, R. J., et al. (2012). Grey matter atrophy in cognitively impaired Parkinson's disease. J. Neurol. Neurosurg. Psychiatry 83, 188-194. doi: 10.1136/jnnp-2011-300828
Melzer, T. R., Watts, R., MacAskill, M. R., Pitcher, T. L., Livingston, L., Keenan, R. J., et al. (2013). White matter microstructure deteriorates across cognitive stages in Parkinson disease. Neurology 80, 1841-1849. doi: 10.1212/WNL. 0b013e3182929f62

Mihaescu, A. S., Masellis, M., Graff-Guerrero, A., Kim, J., Criaud, M., Cho, S. S., et al. (2019). Brain degeneration in Parkinson's disease patients with cognitive decline: a coordinate-based meta-analysis. Brain Imaging Behav. 13, 1021-1034. doi: 10.1007/s11682-018-9922-0

Nakamura, K., and Sugaya, K. (2014). Neuromelanin-sensitive magnetic resonance imaging: a promising technique for depicting tissue characteristics containing neuromelanin. Neural Regen. Res. 9, 759-760. doi: 10.4103/1673-5374. 131583

Nie, K., Zhang, Y., Huang, B., Wang, L., Zhao, J., Huang, Z., et al. (2013). Marked N-acetylaspartate and choline metabolite changes in Parkinson's disease patients with mild cognitive impairment. Parkinsonism Relat. Disord. 19, 329-334. doi: 10.1016/j.parkreldis.2012.11.012

Paez, A. G., Gu, C., Rajan, S., Miao, X., Cao, D., Kamath, V., et al. (2020). Differential changes in arteriolar cerebral blood volume between Parkinson's disease patients with normal and impaired cognition and mild cognitive impairment (MCI) Patients without movement disorder - an exploratory study. Tomography 6, 333-342. doi: 10.18383/j.tom.2020.00033

Pagonabarraga, J., Corcuera-Solano, I., Vives-Gilabert, Y., Llebaria, G., GarcíaSánchez, C., Pascual-Sedano, B., et al. (2013). Pattern of regional cortical thinning associated with cognitive deterioration in Parkinson's disease. PLoS One 8:e54980. doi: 10.1371/journal.pone.0054980

Pagonabarraga, J., Gomez-Anson, B., Rotger, R., Llebaria, G., Garcia-Sanchez, C., Pascual-Sedano, B., et al. (2012). Spectroscopic changes associated with mild cognitive impairment and dementia in Parkinson's disease. Dement. Geriatr. Cogn. Disord. 34, 312-318. doi: 10.1159/000345537

Park, Y. W., Shin, N. Y., Chung, S. J., Kim, J., Lim, S. M., Lee, P. H., et al. (2019). Magnetic resonance imaging-visible perivascular spaces in basal ganglia predict cognitive decline in Parkinson's disease. Mov. Disord. 34, 1672-1679. doi: $10.1002 / \mathrm{mds} .27798$

Pedersen, K. F., Larsen, J. P., Tysnes, O. B., and Alves, G. (2017). Natural course of mild cognitive impairment in Parkinson disease: a 5-year population-based study. Neurology 88, 767-774. doi: 10.1212/WNL.0000000000003634

Pelizzari, L., Di Tella, S., Rossetto, F., Laganà, M. M., Bergsland, N., Pirastru, A., et al. (2020). Parietal perfusion alterations in Parkinson's disease patients without dementia. Front. Neurol. 11:562. doi: 10.3389/fneur.2020.00562

Prasuhn, J., Prasuhn, M., Fellbrich, A., Strautz, R., Lemmer, F., Dreischmeier, S., et al. (2021). Association of locus coeruleus and substantia nigra pathology with cognitive and motor functions in patients with Parkinson disease. Neurology 97, e1007-e1016. doi: 10.1212/wnl.0000000000012444

Ray, N. J., Bradburn, S., Murgatroyd, C., Toseeb, U., Mir, P., Kountouriotis, G. K., et al. (2018). In vivo cholinergic basal forebrain atrophy predicts cognitive decline in de novo Parkinson's disease. Brain 141, 165-176. doi: 10.1093/brain/ awx310

Sampedro, F., Martínez-Horta, S., Marín-Lahoz, J., Pagonabarraga, J., and Kulisevsky, J. (2019). Longitudinal intracortical diffusivity changes in denovo Parkinson's disease: a promising imaging biomarker. Parkinsonism Relat. Disord. 68, 22-25. doi: 10.1016/j.parkreldis.2019.09.031

Scheltens, P., Barkhof, F., Leys, D., Pruvo, J. P., Nauta, J. J., Vermersch, P., et al. (1993). A semiquantative rating scale for the assessment of signal hyperintensities on magnetic resonance imaging. J. Neurol. Sci. 114, 7-12. doi: 10.1016/0022-510x(93)90041-v

Schulz, J., Pagano, G., Fernández Bonfante, J. A., Wilson, H., and Politis, M. (2018). Nucleus basalis of Meynert degeneration precedes and predicts cognitive impairment in Parkinson's disease. Brain 141, 1501-1516. doi: 10.1093/brain/ awy072

Song, S. K., Lee, J. E., Park, H. J., Sohn, Y. H., Lee, J. D., and Lee, P. H. (2011). The pattern of cortical atrophy in patients with Parkinson's disease according to cognitive status. Mov. Disord. 26, 289-296. doi: 10.1002/mds.23477

Sulzer, D., Cassidy, C., Horga, G., Kang, U. J., Fahn, S., Casella, L., et al. (2018). Neuromelanin detection by magnetic resonance imaging (MRI) and its promise as a biomarker for Parkinson's disease. NPJ Parkinsons Dis. 4:11. doi: 10.1038/ s41531-018-0047-3

Tropea, T. F., Xie, S. X., Rick, J., Chahine, L. M., Dahodwala, N., Doshi, J., et al. (2018). APOE, thought disorder, and SPARE-AD predict cognitive decline in 
established Parkinson's disease. Mov. Disord. 33, 289-297. doi: 10.1002/mds. 27204

Tsai, H. H., Tsai, L. K., Lo, Y. L., and Lin, C. H. (2021). Amyloid related cerebral microbleed and plasma $\mathrm{A} \beta 40$ are associated with cognitive decline in Parkinson's disease. Sci. Rep. 11:7115. doi: 10.1038/s41598-021-86617-0

Uchida, Y., Kan, H., Sakurai, K., Arai, N., Kato, D., Kawashima, S., et al. (2019). Voxel-based quantitative susceptibility mapping in Parkinson's disease with mild cognitive impairment. Mov. Disord. 34, 1164-1173. doi: 10.1002/mds. 27717

Uribe, C., Segura, B., Baggio, H. C., Campabadal, A., Abos, A., Compta, Y., et al. (2018). Differential progression of regional hippocampal atrophy in aging and Parkinson's disease. Front. Aging Neurosci. 10:325. doi: 10.3389/fnagi.2018. 00325

Wardlaw, J. M., Smith, E. E., Biessels, G. J., Cordonnier, C., Fazekas, F., Frayne, R., et al. (2013). Neuroimaging standards for research into small vessel disease and its contribution to ageing and neurodegeneration. Lancet Neurol. 12, 822-838. doi: 10.1016/S1474-4422(13)70124-8

Weintraub, D., Dietz, N., Duda, J. E., Wolk, D. A., Doshi, J., Xie, S. X., et al. (2012). Alzheimer's disease pattern of brain atrophy predicts cognitive decline in Parkinson's disease. Brain 135(Pt 1), 170-180. doi: 10.1093/brain/awr277

Weintraub, D., Doshi, J., Koka, D., Davatzikos, C., Siderowf, A. D., Duda, J. E., et al. (2011). Neurodegeneration across stages of cognitive decline in Parkinson disease. Arch. Neurol. 68, 1562-1568. doi: 10.1001/archneurol.2011.725

Wen, M.-C., Ng, A., Chander, R. J., Au, W. L., Tan, L. C. S., and Kandiah, N. (2015). Longitudinal brain volumetric changes and their predictive effects on cognition among cognitively asymptomatic patients with Parkinson's disease. Parkinsonism Relat. Disord. 21, 483-488. doi: 10.1016/j.parkreldis.2015.02.014

Wolters, A. F., van de Weijer, S. C. F., Leentjens, A. F. G., Duits, A. A., Jacobs, H. I. L., and Kuijf, M. L. (2019). Resting-state fMRI in Parkinson's disease patients with cognitive impairment: a meta-analysis. Parkinsonism Relat. Disord. 62, 16-27. doi: 10.1016/j.parkreldis.2018.12.016

$\mathrm{Xu}, \mathrm{Y}$., Yang, J., Hu, X., and Shang, H. (2016). Voxel-based meta-analysis of gray matter volume reductions associated with cognitive impairment in Parkinson's disease. J. Neurol. 263, 1178-1187. doi: 10.1007/s00415-016-8122-3
Zarifkar, P., Kim, J., La, C., Zhang, K., YorkWilliams, S., Levine, T. F., et al. (2021). Cognitive impairment in Parkinson's disease is associated with default mode network subsystem connectivity and cerebrospinal fluid A . Parkinsonism Relat. Disord. 83, 71-78. doi: 10.1016/j.parkreldis.2021. 01.002

Zhan, Z. W., Lin, L. Z., Yu, E. H., Xin, J. W., Lin, L., Lin, H. L., et al. (2018). Abnormal resting-state functional connectivity in posterior cingulate cortex of Parkinson's disease with mild cognitive impairment and dementia. CNS Neurosci. Ther. 24, 897-905. doi: 10.1111/cns.12838

Zheng, D., Chen, C., Song, W., Yi, Z., Zhao, P., Zhong, J., et al. (2019). Regional gray matter reductions associated with mild cognitive impairment in Parkinson's disease: a meta-analysis of voxel-based morphometry studies. Behav. Brain Res. 371:111973. doi: 10.1016/j.bbr.2019.111973

Zhou, C., Guan, X. J., Guo, T., Zeng, Q. L., Gao, T., Huang, P. Y., et al. (2020). Progressive brain atrophy in Parkinson's disease patients who convert to mild cognitive impairment. CNS Neurosci. Ther. 26, 117-125. doi: 10.1111/cns. 13188

Conflict of Interest: The authors declare that the research was conducted in the absence of any commercial or financial relationships that could be construed as a potential conflict of interest.

Publisher's Note: All claims expressed in this article are solely those of the authors and do not necessarily represent those of their affiliated organizations, or those of the publisher, the editors and the reviewers. Any product that may be evaluated in this article, or claim that may be made by its manufacturer, is not guaranteed or endorsed by the publisher.

Copyright $(2022$ Hou and Shang. This is an open-access article distributed under the terms of the Creative Commons Attribution License (CC BY). The use, distribution or reproduction in other forums is permitted, provided the original author(s) and the copyright owner(s) are credited and that the original publication in this journal is cited, in accordance with accepted academic practice. No use, distribution or reproduction is permitted which does not comply with these terms. 